\title{
Visual lobe dimensions and search performance for targets on a competing homogeneous background
}

\author{
A. J. COURTNEY and H. S. CHAN \\ University of Hong Kong, Hong Kong
}

\begin{abstract}
The relationship between visual lobe area and search performance has been demonstrated by previous researchers under a variety of experimental conditions and using different measures of lobe area. The relationship has not been as highly significant as might be expected, given the obvious importance of lobe area for search performance. Using uniform stimulus material for lobe size and search performance tests, specifying viewing distance, controlling the presentation of search task material, and eliminating some extreme search strategy options, we obtained a very highly significant correlation between lobe area and search performance for 54 Cantonese Chinese subjects. This result was obtained in spite of the subjects' homogeneity with regard to age and near foveal acuity. An unexpected result was that, relative to the horizontal lobe dimension, the vertical dimension was highly significantly related to lobe area and to search performance.
\end{abstract}

Although the visual lobe (or conspicuity area) has been widely used in models of visual search, there is no agreed definition, or any agreed method of measurement, of the lobe. In fact, the definition will depend upon the method of measurement used. The reason for measuring lobe size is usually to obtain a single parameter that determines some aspect of performance in visual search tasks. Lamar (1960) produced equations, later refined by Taylor (1961), to define the visual detection lobe for use in an exponential glimpse model of visual search. He was concerned with air-to-sea search, and his visual detection lobe equations, which defined the just detectable value of target/background contrast, were a function of target size and of angle off the visual axis. Even though Lamar was concerned with simple detection involving a single target on a uniform background, his visual detection lobe equations have been very influential in later efforts to model more complex search situations. Although visual lobes for simple detection can be predicted quite well, the situation is not so clear when the search is for more complex objects or when higher-order decisions are required. Recently, Engel (1971) proposed that the visual lobe (conspicuity) area of an object can be used as a measure of the visual conspicuity of the relevant object, and Engel (1977) defined the conspicuity area as the retinal field in which an object is capable of being noticed during a single eye pause. Engel (1977) determined conspicuity area by using only one target (known beforehand by the subject) against a background pattern; therefore, the task was one of detecting in eccentric vision "something" different from the background. The visual lobes determined in the experiment reported here were similar in concept to Engel's conspicuity area but were calculated in a differ-

The authors' address is Department of Industrial Engineering, University of Hong Kong, Pokfulam Road, Hong Kong. ent way. Bloomfield and Howarth (1969) and Bloomfield et al. (1974) developed equations for the relationship between target conspicuity and search performance which were summarized by Bloomfield (1975a) as $t \propto 1 / \theta^{2}$ and $t \propto 1 / D^{2}$, where $t=$ mean search time, $\theta=$ the extent into the periphery to which discrimination can be made, and $D=$ the discriminability of the target. In one form or another, the visual lobe area plays a very important role in models of visual search (for recent examples, see Brown, 1979, and Overington, 1979).

During search, a series of eye fixations averaging 200-300 msec in duration will occur over the visual scene (Megaw \& Bellamy, 1979). For static displays larger than the foveal area, the amount of information that may be obtained during each fixation will depend upon the peripheral acuity of the observer and upon the physical characteristics of the target and the area being searched. The assumptions here are that search time will depend primarily on the number of fixations required to find the target, which in turn will depend on the visual lobe area for that target. Number of fixations does indeed correlate highly with search time (Megaw \& Bellamy, 1979), even though many other variables may be involved. (For a useful discussion of search models, see Megaw and Bellamy, 1979.) It is not surprising, then, that the relationship between visual lobe area (or some measure proportional to lobe area) and search performance has been established for real targets with trained observers (Leachtenauer, 1978) and for laboratory tasks (Bellamy \& Courtney, 1981; Erickson, 1964; Johnston, 1965). What is surprising is that most of the correlations were not as highly significant as might have been expected, given the obvious importance of lobe area for search performance. Many factors may interact with lobe area in determining search performance. The nature of the search task and the search material used are important, as are 
the motivation and attitude of the subjects (Ikeda \& Takekeuchi, 1975). The strategy adopted by the searcher may also have a profound effect on search time. Theoretically, a systematic "efficient" strategy would require only half the time required by a random strategy (Howarth \& Bloomfield, 1968). In practice, however, regardless of the original intent of the searcher, it may be difficult or even impossible to adopt an efficient strategy (Bloomfield, 1975a). Bloomfield (1975b) and Megaw (1979) provide useful discussions of the factors that affect search performance in practical situations.

An addditional factor may be the nature of the measurements used to assess lobe area. Because of the large number of readings required to fully map the lobe area (Chaikin, Corbin, \& Volkmann, 1962), only one or two axes have been used to take readings and a wide variety of techniques have been employed. For example, Erickson (1964) measured peripheral acuity by exposing a Landholt $\mathrm{C}$ for $1.5 \mathrm{sec}$ in a blank white field at $3.6^{\circ}, 4.8^{\circ}$, and $6^{\circ}$ to the right of binocular fixation. Correlations between search time and peripheral acuity were not good for individual sessions (only two out of the six sessions achieved $p<.05 ; r=-.669$ for Session 2 and $r=-.497$ for Session 5), but average results were significant at .01 $(r=-.642)$. By careful post hoc analysis, choosing only the $3.6^{\circ}$ and $4.8^{\circ}$ eccentricities and only part of the search data, a coefficient of $-.8(p<.001)$ was obtained. $\mathrm{Cu}-$ riously, for the acuity test, 10 out of the 16 subjects produced better results, in terms of percentage of targets correct, at some of the more distant eccentricities than at some of the nearer eccentricities. Johnston (1965) used a white Landholt $C$ against a plain black background and a $0.75-\mathrm{sec}$ exposure to determine $75 \%$ monocular threshold points for a Landholt $\mathrm{C}$ on each of the four meridians (temporal, nasal, superior, and inferior) in turn. Correlations for $\log$ transformed visual field area and $\log$ search times were significant at $.05(r=-.297)$ and .01 $(r=-.484)$, respectively, for different search tasks. Although visual field size was significantly correlated with the temporal meridian measure, the correlations were too low to be reliable and a recommendation was made that thresholds on the four meridians were necessary to determine visual field (lobe) size. With no mention of exposure time, Leachtenauer (1978) used a Landholt C against a plain background and a technique similar to that of Johnston (1965) to determine monocular thresholds on four meridians for each eye. These two monocular fields were then combined to provide visual field area in square degrees. Log field area was found to correlate with various search task scores (or arc sin transformed scores) at the .05 level $(r=.884)$. Bellamy and Courtney (1981), using a four-meridian measure of lobe area based on the magnitude of errors made by their subjects, found a correlation significant at $.01(r=-.920)$ between lobe area and search performance. Subjects of widely different ages and from different backgrounds were used in an experiment by Courtney (1984). He showed that by using a simplified form of lobe calculation, where the boundary was placed at an eccentricity proportional to the number of correct responses on each meridian, the correlation between lobe area and search performance was significant at .0005 $(r=-.885)$. However, when all the subjects above $\mathbf{3 0}$ years of age were excluded from the analysis, the correlation was significant at the .005 level $(r=-.690)$. Courtney and Chan (1985), testing a shorter search task, found that lobe area determined by a method similar to, but quicker than, that used by Courtney (1984) correlated at the $.01(r=.400)$ level with search time. Courtney and Chan suggested that, due to the nature of the search task, search strategy may have been an important factor in their experiment.

The present experiment was aimed at establishing the relationship between lobe area and search performance under conditions of more careful control than those in the previous work of Bellamy and Courtney (1981), Courtney (1984), and Courtney and Chan (1985).

\section{METHOD}

The study consisted of several parts. Each subject attended one session of about $1 \mathrm{~h}$. The near and far foveal acuity of each subject was tested using a Bausch and Lomb orthorator. Test material was presented tachistoscopically to measure lobe size, and following a 5-min rest, a card-sorting task was given to assess search performance.

\section{Subjects}

Fifty-four subjects, 47 males and 7 females, participated in the study. They were all Hong Kong Chinese undergraduates in the Department of Industrial Engineering of the University of Hong Kong. Their ages ranged from 18 to 24 years, with a median of 21 .

\section{Visual Lobe Measurement}

A method very similar to that described by Bellamy and Courtney (1981) was used, but there was only one target type $(v)$ and stimulus presentation followed a modified format.

Stimulus cards were prepared by offset printing to fit a two-field tachistoscope (Electronic Development's portable two-field model); each card contained one target character $(v)$ against a background of 677 regularly spaced $x$ s. Figure 1 shows part of a stimulus card.

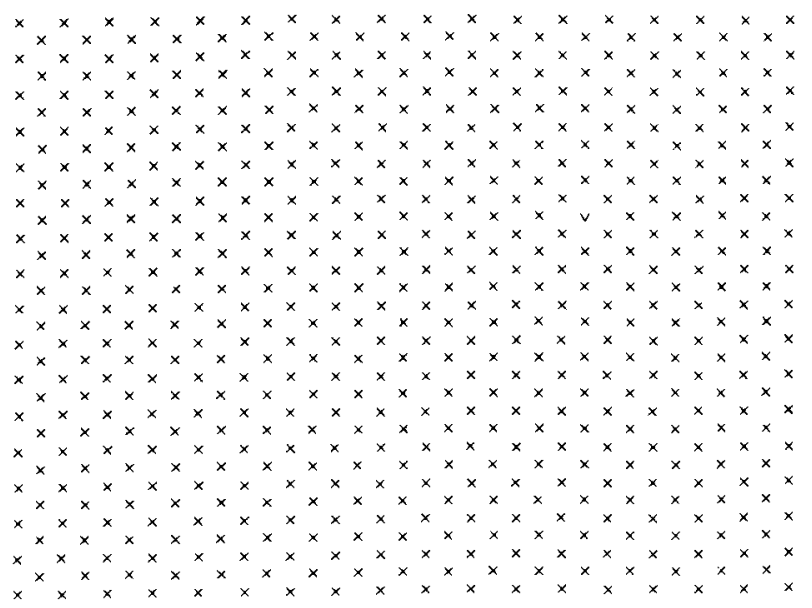

Scale: $0,100 \mathrm{~min}$ arc.

Figure 1. Stimulus material used for lobe size and search performance determination. Note: Only part of the stimulus field is shown here. 
The tachistoscope had a $176 \times 104 \mathrm{~mm}$ active viewing area, presented at $508 \mathrm{~mm}$ and subtending $19.7^{\circ}$ horizontally and $11.7^{\circ}$ vertically. The characters on the card were dimensioned as shown in Figure 2, subtended $11.2^{\prime} \times 11.2^{\prime}$ of arc, and were separated center to center by $8.6 \mathrm{~mm}\left(58.2^{\prime}\right.$ of arc) horizontally and $6.6 \mathrm{~mm}$ (44.7' of arc) vertically. The fixation point, which was on the blank second field of the tachistoscope, coincided with the center of the stimulus array at a point not occupied by an $x$. The stimulus and fixation field luminances were $75 \mathrm{~cd} / \mathrm{m}^{2}$. The circuit was connected so that the fixation field was presented continuously until a pushbutton was operated to expose the stimulus. Timing accuracy was within $2.5 \%$ of the indicated time interval. The single $v$ target on each card was located by replacing an $x$ on either the horizontal or vertical axis passing through the fixation point. Targets were placed at eight locations to the right and left of the fixation point: 87.3', 145.5', $203.7^{\prime}, 261.9^{\prime}, 320.1^{\prime}, 378.3^{\prime}, 436.5^{\prime}$, and 494.7' of arc; and at seven locations above and below the fixation point: $67.1^{\prime}, 111.7^{\prime}$, $156.4^{\prime}, 201.1^{\prime}, 245.8^{\prime}, 290.6^{\prime}$, and 335.2' of arc.

Each subject attended one session, during which each of the 30 stimulus cards was presented twice tachistoscopically for $250 \mathrm{msec}$. Presentation was divided into two parts: after six practice trials, all the cards were presented once in random order; after a 3-min rest, the cards were presented for a second time in random order. The subject initiated the stimulus presentation him- or herself after
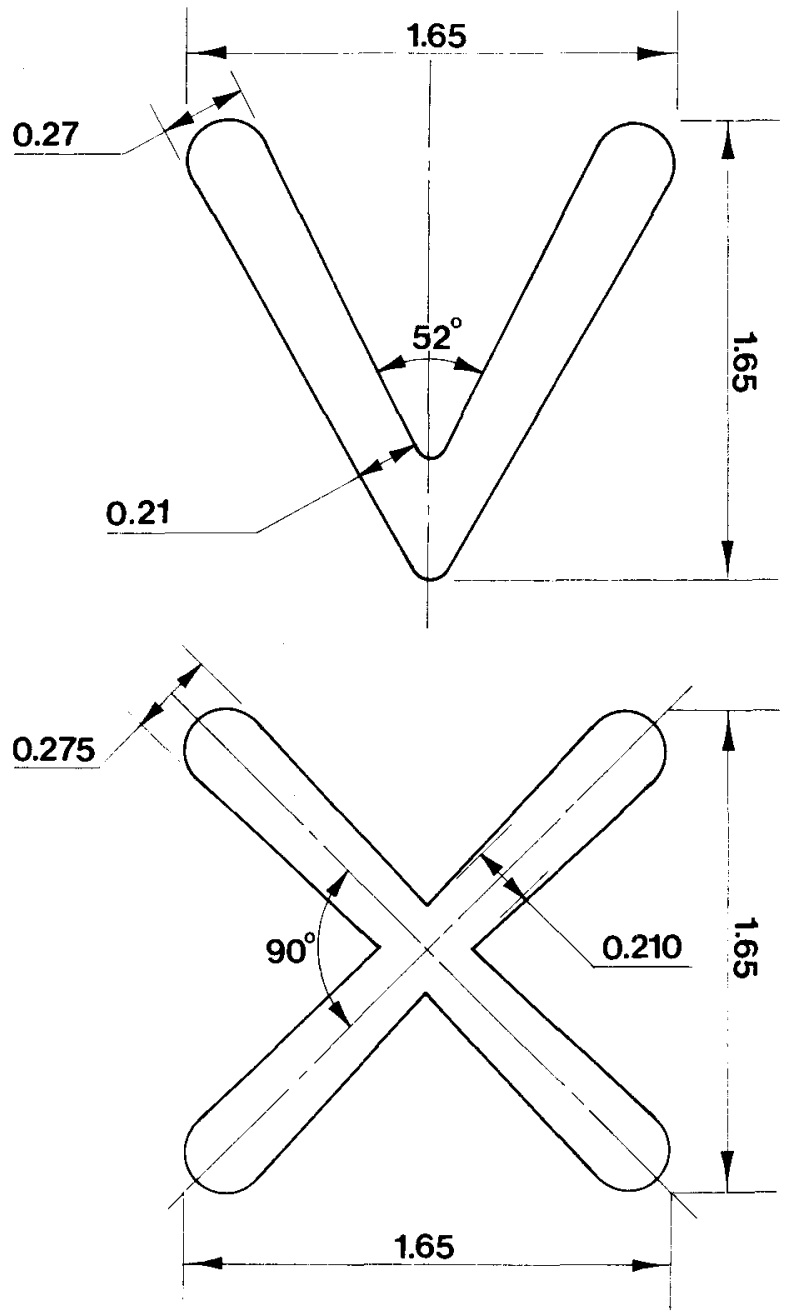

Figure 2. Dimensions of target $(v)$ and background $(x)$ characters used for lobe size and search performance stimulus material. fixating a spot on the second field of the tachistoscope. Immediately after each presentation, the subject gave an estimate of target location on a specimen card showing numbered points on the vertical and horizontal meridians. The subject was required to indicate a location even if he or she did not see a target. Thus, for each target position, the subject gave an estimated location from which an error score could be calculated.

\section{Search Time Measurement}

Stimulus material for the search cards was similar to that used for lobe measurement. The cards were $153 \times 77 \mathrm{~mm}$ with characters of $1.65 \times 1.65 \mathrm{~mm}$, as shown in Figure 2. Two targets were used, $<$ and $>$; these were the $v$ target shown in Figure 2, but pointing to the left or right. These targets were sufficiently similar that no significant target conspicuity difference would be expected. Each card contained only one target, which was randomly positioned on a stratified basis so that targets appeared with equal probability in each cell of an imaginary matrix of 12 equal-sized cells superimposed on the card. No targets were placed on the outside edges of the cards, so that all the targets were completely surrounded by $x$ s. Two packs of 24 cards each were prepared, with each target type appearing on 12 cards in each pack.

A simple device called the card changer was used to present the cards. It was a $36 \times 32 \mathrm{~cm}$ white-painted board inclined $9^{\circ}$ from vertical, so that the bottom of the board was nearer than the top to the subject. This board had a $15.3 \times 7.7 \mathrm{~cm}$ rectangular opening at the center, showing a white background with a fixation cross in the center. The subject held a small control box with two buttons. When the first button was pressed, a stimulus card was released and within $40 \mathrm{msec}$ dropped into view in front of the fixation cross. The card's dropping into view started an electronic timer. The second button stopped the timer and dropped the card from view.

A 5-min rest was given after the lobe measurements were finished and then the card changer trials were performed. The subject was seated so that he/she could view the card changer comfortably with his $/$ her eyes at about $75 \mathrm{~cm}$ from the fixation cross. At this distance the background characters and targets subtended $7.56^{\prime} \times 7.56^{\prime}$ of arc. The subject was instructed to keep his/her eyes at a specified distance $(75 \mathrm{~cm})$ from the card changer and a distance guide was provided. All subjects obeyed this instruction very well. The subject fixated the center cross and, when ready, pressed the first button to display a stimulus card. When the target was located, the subject pressed the second button and reported whether the target was a left- $(<)$ or right- $(>)$ pointing target. Each subject had six practice trials followed by the presentation of one pack of 24 cards. After a 1-min rest, the second pack of 24 cards was presented, using exactly the same procedure as for the first pack.

\section{RESULTS}

\section{Lobe Dimensions}

The method described by Courtney (1984) and Courtney and Chan (1985), which is based on the average number of "correct" responses made on each axis, was used for calculating relative lobe size for each subject. A response was correct when the error score was no greater than two target locations (i.e., 116.4' of arc horizontal and $89.4^{\prime}$ of arc vertical). The error score for each response was the difference between actual and estimated target locations, expressed in minutes of arc. The locations adjacent to the fixation point were not used for target presentation and it was assumed that each subject could detect targets at these innermost locations on all four meridians. Previous results (Bellamy \& Courtney, 1981) 
had shown that even subjects with small lobe sizes would rarely miss targets adjacent to the fixation point. The product of the average number of correct responses for each meridian and center-to-center target separation (58.2' of arc horizontal, 44.7' of arc vertical) was taken as the lobe boundary for that meridian. This method of calculation gives an operational definition for the lobe boundary that has been shown by Courtney and Chan (1985), using similar stimulus material, to correlate highly with the lobe boundary established by a more complex method of calculation that is based on linear regression of transformed error scores. (The simpler method of calculation provides an operational definition suitable for the type of material used here, but may not be appropriate for different types of target and background combinations.) The choice of two target locations to define a correct response was consistent with the $115^{\prime}$ of arc used by Bellamy and Courtney (1981) which was based on the average error (over all their subjects) made at a subjective confidence rating of 50 (on a scale from 0 to 100). The left and right meridians were combined to produce an average for the horizontal axis $(\mathrm{H})$, and the top and bottom meridians were combined to give an average for the vertical axis (V). For example, if a subject had averages of 4, 6.5, 4.5 , and 4 correct responses on the top, bottom, left, and right meridians, the top and bottom boundaries were $4 \times 44.7=178.8^{\prime}$ of arc and $6.5 \times 44.7=290.5^{\prime}$ of arc, giving $\mathrm{V}=0.5(178.8+290.6)=234.7^{\prime}$ of arc, and similarly $H=0.5(4.5+4) 58.2=247.3^{\prime}$ of arc. Lobe area in square minutes of arc was computed for each subject, using the formula Area $=2 \times \mathrm{V} \times \mathrm{H}$, which provided the area encompassed by a rhombus-shaped visual lobe similar to the four-sided kite-shaped lobe area given by Johnston's (1965) formula.

For the 54 subjects, vertical dimensions (V) ranged from $67^{\prime}$ to $234.5^{\prime}$ of arc, with a median of $156.3^{\prime}$ of arc; horizontal dimensions ranged from $130.9^{\prime}$ to $349.2^{\prime}$ of arc, with a median of $203.2^{\prime}$ of arc; and area ranged from $20,466.8$ to 148,141 square minutes of arc, with a median of $63,674.5$ square minutes of arc. Very high product-moment correlations were found between visual lobe area and vertical length (V) $(r=.818, p<.0005$, $d f=52$ ) and between lobe area and horizontal length $(\mathrm{H})$ $(r=.493, p<.0005, d f=52)$, but $\mathrm{V}$ and $\mathrm{H}$ were not significantly correlated $(p>.05)$. A $t$ test showed that top and bottom meridians were equal, and that right and left meridians were equal $(p>.1)$, indicating vertical and lateral symmetry in lobe shape based on four meridians. Horizontal length $(\mathrm{H})$ was significantly greater than vertical length ( $t$ test, $p<.0001)$, which was reflected in the fact that the ratio $\mathrm{V} / \mathrm{H}$ was less than 1 for 47 out of the 54 subjects. The 7 subjects that had $\mathrm{V} / \mathrm{H}$ ratios greater than 1 all had above-average vertical lengths $\left(>150.5^{\prime}\right.$ of arc) and below-average horizontal lengths ( $<220.6^{\prime}$ of arc). A significant product-moment correlation $(p<.005)$ between lobe area and $\mathrm{V} / \mathrm{H}$ ratio suggested that, as area increased, vertical length increased proportionally more than horizontal length.

\section{Search Performance}

Mean card-sorting time for the first pack was $3.599 \mathrm{sec}$ per card, with a standard deviation of $2.07 \mathrm{sec}$ per card and a range from 1.669 to $13.920 \mathrm{sec}$ per card. The mean for the second pack was $3.274 \mathrm{sec}$ per card, with a standard deviation of $1.542 \mathrm{sec}$ per card and a range from 1.502 to $9.634 \mathrm{sec}$ per card. A $t$ test showed that firstpack search times were significantly slower than secondpack times $(p<.025$, one-tail). The product-moment correlation between first- and second-pack search times was very high $(r=.8356, p<.0005, d f=52)$.

A $t$ test on the search times for the two types of targets ( $>$ and $<$ ) showed no significant difference $(p>.025)$, justifying the assumption of equal target conspicuity. The 12 cells used to locate the random targets are shown in Figure 3. Analysis of variance on search times showed that, for the 12 cells, there was no significant row effect but there was a significant column effect and a significant row $\times$ column interaction $(p<.005)$. Further analysis using Duncan's multiple range test showed that cells $\mathrm{D}, \mathrm{H}$, and I had significantly longer search times than other cells $(p<.01)$.

\section{Visual Lobe Dimensions and Search Performance}

Table 1 shows the correlation matrix for the lobe dimensions and search performance. Lobe area correlated very highly significantly with first- and second-pack search performance times and with the average of first- and secondpack times $(p<.0005)$. Visual search performance correlated highly with vertical lobe length $(p<.005)$, but the best correlation with horizontal length was at the 0.025 level for second-pack search times.

\section{Acuity Effects}

Only one subject had near acuity less than $8(20 / 25$ Snellen notation) on the Bausch and Lomb orthorator, and his score was 7 . Scores for far acuity ranged from 4 to 12 on the orthorator and these did not correlate significantly with near acuity $(p>.05)$. Near and far acuity did not correlate significantly with any of the lobe dimensions or with search performance $(p>.05)$.

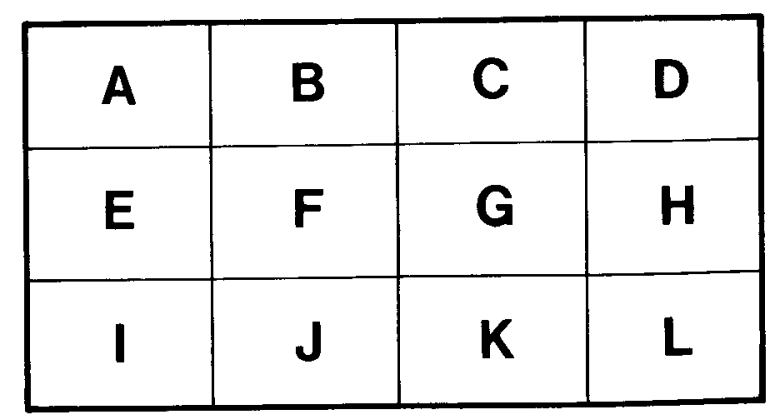

Figure 3. The 12 cells used to locate random targets on the search task cards. 
Table 1

Product-Moment Correlations Between Card Search Times

(Pack 1, Pack 2, and Average of Packs 1 and 2)

and Lobe Dimensions (Horizontal, Vertical, and Area)

\begin{tabular}{|c|c|c|c|c|c|c|}
\hline \multirow[b]{3}{*}{ Search Times } & \multicolumn{6}{|c|}{ Lobe Dimensions } \\
\hline & \multicolumn{2}{|c|}{ Vertical (V) } & \multicolumn{2}{|c|}{ Horizontal $(\mathrm{H})$} & \multicolumn{2}{|c|}{ Area $(2 \mathrm{VH})$} \\
\hline & $r$ & $p$ & $r$ & $p$ & $r$ & $p$ \\
\hline Pack 1 & -.431 & .005 & -.193 & n.s. & -.442 & .0005 \\
\hline Pack 2 & -.410 & .005 & -.271 & .025 & -.468 & .0005 \\
\hline Average & -.440 & .005 & -.236 & .05 & -.472 & .0005 \\
\hline
\end{tabular}

Note-n.s. $=p>.05$.

\section{DISCUSSION}

Lobe area based on four meridians was symmetrical about the fixation point, with the horizontal length greater than the vertical length. This result agrees with previous work (Chaikin et al., 1962), although for similar stimulus material Bellamy and Courtney (1981) found no difference between horizontal and vertical axes. The very high correlations found between lobe area and vertical and horizontal length suggest that area could be estimated from measurements on one axis only. However, the lack of correlation between vertical and horizontal length indicates that the ratio between vertical and horizontal lengths was not consistent across subjects. This ratio, which was less than 1 for most subjects, correlated significantly with lobe area. This correlation, together with the very high correlation between area and vertical length, indicated that for these subjects vertical length varied with lobe area proportionately more than did horizontal length, and variations in lobe area appeared to be due more to variations in vertical length than to variations in horizontal length. The special importance of vertical length was not expected on the basis of earlier work (Bellamy \& Courtney, 1981; Johnston, 1965), and the phenomenon calls for further investigation.

There were some important differences between the search performance measured in the present experiment and that obtained by Bellamy and Courtney (1981), Courtney (1984), and Courtney and Chan (1985). The subjects in the present experiment did not sort cards but viewed the cards in a card changer, so that no movement or manipulation times were involved and viewing distance was held relatively constant. The targets used here ( $>$ and $<$ ) were of equal conspicuity and could appear on any part of the stimulus cards except the outermost locations. Bellamy and Courtney (1981) and Courtney (1984) used targets of different conspicuities, which allowed the subjects to adopt the extreme strategy of searching for only the easier target, and if it could not be found, assuming that the card contained the more difficult target. Courtney and Chan (1985) used only one target, with the cards sorted on the basis of target location (i.e., left or right of an imaginary center line). Their subjects could adopt the extreme strategy of searching only one side of a card, and if the target could not be found, assuming that it must be on the other side. One subject with small lobe dimensions and fast search time admitted to following such a strategy. The two targets used in the present experiment were of equal conspicuity, so it would be difficult for a subject to adopt the type of extreme strategy noted above; nevertheless, there was no compulsion concerning search strategy, and without monitoring eye movements, we cannot know the variety of strategies adopted by the subjects. None of the subjects made mistakes in reporting the targets, so it seems reasonable to assume that they actually located most targets. There was a learning effect between the first and second packs, as expected from previous work, and search times for the first and second packs were very highly significantly related, as in previous work (Bellamy \& Courtney, 1981). Targets were located on the stimulus card so as to appear with equal probability in each cell of a matrix of 12 imaginary cells. A significant column effect was found, but no row effect, and targets at the top right and bottom left cells took significantly longer to find than targets at other locations. These effects may have been due to search strategies that neglected some parts of the cards, but without knowledge of scan paths we cannot be certain. Enoch (1959) obtained a similar finding with a nonhomogeneous field for search on aerial maps, but with the neglected portion at the top left. Bloomfield (1972) found that longer search times were required for targets lying on the boundary than for those at central locations.

Search time was highly sensitive to lobe area and to vertical lobe dimensions, but not to horizontal lobe dimensions. The very high correlation between search time and area $(p<.0005)$ suggests that lobe area was a good predictor of search performance when both area and search performance were measured using the same stimulus material and that search performance provided a good measure of relative lobe size. Bellamy and Courtney (1981) obtained a high reliability coefficient for a cardsorting task using stimulus material similar to that used in the present experiment. The relationship may be affected by perceptual learning and needs to be related to amount and type of practice. However, encouraging results were obtained by Leachtenauer (1978), who found that over a period of about 1 year his subjects maintained their rank-order positions for visual lobe area despite differences in intervening training and work experience. The good relationship between lobe area and search time was obtained in the present experiment in spite of a number of factors that militated against such a result. The subjects were all young ( $<30$ years of age), and with the exception of one subject with a score of 7 , all had reasonable near foveal acuity ( $\geq 8$, Bausch and Lomb orthorator). Courtney (1984) found that for subjects below age 30 with near acuity of at least 8 , card-sorting time was not so highly significantly correlated with lobe area as it was for a group of subjects less homogeneous in age and acuity.

The high correlation of search time with vertical lobe dimension $(p<.005)$ but not with horizontal dimension was surprising in view of the importance of horizontal 
dimension suggested by Bellamy and Courtney (1981) and by the generally accepted importance of measurements made on the horizontal lobe axis (Erickson, 1964; Gallwey, 1982). The vertical lobe dimension here played a more significant part in determining lobe area and search performance than did the horizontal dimension, and a provocative suggestion is that ethnic and cultural factors may have played an important role. The subjects used here were Cantonese Chinese, their first language was not English, and their reading habits may not have conformed completely to the left-to-right Western pattern. Courtney (1984) and Courtney and Chan (1985), also using Cantonese Chinese subjects, found that the vertical lobe dimension was at least as significant as, and in some cases more significant than, the horizontal dimension in correlation with card-sorting times.

\section{REFERENCES}

Bellamy, L. J., \& Courtney, A. J. (1981). Development of a search task for the measurement of peripheral acuity. Ergonomics, 24, 497-509.

BLOOMfield, J. R. (1972). Visual search in complex fields: Size differences between target disc and surrounding discs. Human Factors, 14, 139-148.

BLOOMFIELD, J. R. (1975a). Studies on visual search. In J. G. Fox \& C. G. Drury (Eds.), Human reliability and quality control (pp. 3143). London: Taylor \& Francis.

Bloomfield, J. R. (1975b) Theoretical approaches to visual search. In J. G. Fox and C. G. Drury (Eds.), Human reliability and quality control (pp. 19-29). London: Taylor \& Francis.

Bloomfield, J. R., BeckWith, W. E., Emerick, J., Marmurek, H. H., TEI, B. E., \& TRAUB, B. H. (1974). Visual search with embedded targets (ARI Tech. Rep. TR-78-TN8). Alexandria, VA: U.S. Army Research Institute for the Behavioral and Social Sciences.

BloOMfield, J. R., \& HowarTH, C. I. (1969). Testing visual search theory. In H. W. Leibowitz (Ed.), Image evaluation (pp. 203-214). Proceedings of NATO Advisory Group on Human Facttors, Munich.

Brown, M. B. (1979). Mathematical approaches to modelling visual search. In J. N. Clare \& M. A. Sinclair (Eds.), Search and the human observer (pp. 126-131). London: Taylor \& Francis.
Chaikin, J. D., Corbin, H. H., \& Volkmann, J. (1962). Mapping a field of short-time visual search. Science, 138, 1327-1328.

Courtney, A. J. (1984). A search task to assess visual lobe size. $H u$ man Factors, 23, 289-298.

Courtney, A. J., \& Chan, H. S. (1985). Simple measures of visual lobe size and search performance. Ergonomics, 28, 1319-1332.

ENGEL, F. L. (1971). Visual conspicuity, directed attention and retinal locus. Vision Research, 11, 563-576.

ENGEL, F. L. (1977). Visual conspicuity, visual search and fixation tendencies of the eye. Vision Research, 17, 95-108.

ENOCH, J. M. (1959). Effect of the size of a complex display upon visual search. Journal of the Optical Society of America, 49, 280-286.

ERICKSON, R. A. (1964). Relation between visual search time and peripheral visual acuity. Human Factors, 6, 165-178.

GallWEY, T. J, (1982). Selection tests for visual inspection on a multiple-fault type task. Ergonomics, 25, 1077-1092.

HowarTH, C. I., \& BloomfiELD, J. R. (1968). Towards a theory of visual search. In AGARD Conference Proceedings (No. 41). Brussels: AGARD.

IKEDA, M., \& TAKEKEUCHI, T. (1975). Influence of foveal load on the functional visual field. Perception \& Psychophysics, 18, 255-260.

JoHNSTON, D. M. (1965). Search performance as a function of peripheral acuity. Human Factors, 7, 528-535.

LAMAR, E. S. (1960). Operational background and physical considerations relative to visual search problems. In A. Morris \& E. P. Horne (Eds.), Visual search techniques (pp. 1-9). Washington, DC: National Academy of Sciences.

Leachtenauer, J. C. (1978). Peripheral acuity and photointerpretation performance. Human Factors, 20, 537-551.

Megaw, E. D. (1979). Factors affecting visual inspection accuracy. Applied Ergonomics, 10, 27-32.

Megaw, E. D., Bellamy, L. J. (1979). Eye movements and visual search. In J. N. Clare \& M. A. Sinclair (Eds.), Search and the human observer (pp. 65-73). London: Taylor \& Francis.

OvERINGTON, I. (1979). The current status of mathematical modelling of threshold functions. In J. N. Clare \& M. A. Sinclair (Eds.), Search and the human observer (pp. 114-125). London: Taylor \& Francis.

TAYLOR, J. H. (March, 1961). Contrast thresholds as a function of retinal position and target size for the light-adapted eye (Scripps Institute of Oceanography Ref. 61-10). San Diego: University of California Visibility Laboratory.

(Manuscript received April 24, 1985; revision accepted for publication March $31,1986$. 